MATEC Web of Conferences 22,01022 (2015)

DOI: $10.1051 /$ matec conf/ 20152201022

(C) Owned by the authors, published by EDP Sciences, 2015

\title{
Data Transmission Strategy for Wireless Sensor Network Based on Vir- tual MIMO
}

\author{
Chenwei Feng* \\ School of Opto-electronic and Communication Engineering, Xiamen University of Technology, Xiamen, Fujian, \\ China
}

\begin{abstract}
Virtual multiple input multiple output (MIMO) technology has been widely researched in the aspect of energy conservation for wireless sensor network (WSN) communication. In this this paper, data transmission for the entire network based on virtual MIMO WSN structure is studied, combined with clustering and multi-hop transmission mode. Each cluster head $(\mathrm{CH})$ is formed according to the clustering algorithm, and the data is transmitted to the adjacent $\mathrm{CH}$ by cooperative communication mode. The circuit energy consumption and the transmission energy consumption are both considered in our model, so the minimum inter-cluster transmission energy consumption is obtained, and the optimal path to the sink node is obtained. The proposed strategy does not need the receiver's collaboration to avoid the complexity of the receiver; meanwhile more energy consumption of the network is saved from the perspective of the network life cycle. The simulation results show that the proposed strategy can reduce more energy consumption and prolong the network life cycle.
\end{abstract}

Keywords: virtual MIMO; WSN; cooperative communication; multi-hop transmission

\section{INTRODUCTION}

In recent years, virtual MIMO technology has been paid increasing attention in the industry due to its high energy efficiency [1]. The virtual MIMO technology has been applied in WSN to reduce energy consumption and increase reliability. In the virtual MIMO network, multiple sensor nodes cooperatively send and receive data. Virtual MIMO technology in the aspect of long-distance communication of WSN can reduce the transmission energy consumption, but increase the circuit energy consumption as the number of transceivers increases, and the total energy consumption as well. The virtual MIMO technology is first introduced to WSN in this paper [2], which shows that over certain distance ranges the total energy consumption can be reduced compared with the single input single output (SISO) transmission mode; the data is coded at transmitter side using the space-time block code (STBC); the influence on energy consumption over constellation size or distance is analyzed, but the energy consumption is still larger without using multi-hop transmission.

Combining with multi-hop routing and scheduling, this paper [3] proposes a cross-layer collaborative transmission to reduce energy consumption and improve the end-to-end performance of the network. The energy consumption is optimized in this paper [4] based on virtual MIMO technology by optimizing the constellation size, and the first sensor node death time is delayed. Although multi-hop transmission mode is adopted in this paper [2-4], the method of energy consumption optimization is enumeration without flexibility. This paper [5] proposes a routing scheme

*Corresponding author: cwfeng@,xmut.edu.cn based on ant colony algorithm to reduce the network energy consumption through analyzing the algorithm of Low Energy Adaptive Clustering Hierarchy $(\mathrm{LEACH})$, but the total energy consumption is not exact without considering the circuit energy consumption.

This paper proposes a data transmission strategy for WSN based on virtual MIMO by considering the total energy consumption consisting of transmission energy consumption and circuit energy consumption comprehensively. The data is forwarded to the adjacent $\mathrm{CH}$ collaboratively, and the minimum inter-cluster energy consumption can be obtained by the energy model. Finally, to save more energy and prolong the network life cycle, the data reach to the sink node through the optimal path based on the simple and practical routing algorithm Dijkstra.

This paper is organized as follows: the background and the related work are introduced in Section 1; the system model and the proposed strategy are described detailedly in Section 2; the energy model is illustrated in Section 3; the simulation results are shown in Section 4; finally, concluding remarks are given in Section 5 .

\section{SYSTEM STRATEGIES}

\subsection{WSN model based on virtual MIMO}

Data transmission in traditional WSN achieved by only a transmitter and a receiver, known as SISO, will cost large energy consumption when the transmission distance is long. Cooperative virtual MIMO commu- 


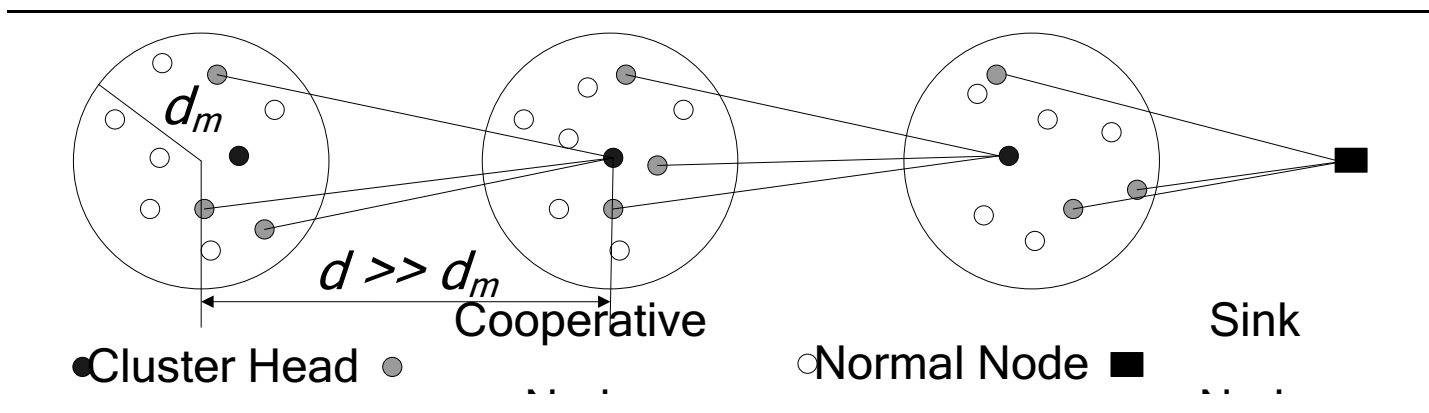

Figure 1. Virtual MIMO model of cooperative communication nication has better performance than SISO communication in the aspect of energy conservation, especially for long distance transmission [2]. This is because multiple sensor nodes are integrated in virtual cooperative MIMO communication, which allows multiple single antenna sensor nodes to form an antenna array of MIMO system.

The cooperative virtual MIMO communication model based on cluster [6] is shown in Figure 1, in which cluster can be formed by a certain clustering algorithm such as LEACH. In general, the average inter-cluster distance $d$ (approximating $d$ with the distance between the centers of the two clusters) need to be larger than the radius of the cluster $d_{m}$, that is, $d>d_{m}$.

In the process of cooperative transmission, the transmission cluster consists of $M_{t}$ cooperative sensor nodes, which form a virtual antenna array with $M_{r}$ sensor nodes at the receiver side. Both transmitters and receivers sides need to work collaboratively in existing virtual MIMO schemes, but most of which only mention the virtual communication solution without considering how to realize the synergy at the receiver side [7]. In order to reduce the complexity of the data processing at receiver side, this paper only considers multiple input single output (MISO) mode, namely only the $\mathrm{CH}$ receive data at the receiver side.

\subsection{Data transmission strategy}

In order to transfer the data from one sensor node to the sink node, the cluster where this sensor node located selects some proper cooperative sensor nodes based on the inter-cluster distance, and transmits the data to the adjacent $\mathrm{CH}$ through virtual MISO. When the data is received and decoded correctly by the $\mathrm{CH}$, the receiver side continues to forward the data to the next $\mathrm{CH}$ in the same way until the data reach to the sink node. Combining with the model shown in Figure 1 , the specific procedure is as follows:

(1) Clustering

Without loss of generality, $\mathrm{CH}$ is formed by LEACH, in which each sensor node generates a number between $0 \sim 1$ randomly. If the number is smaller than the given threshold $T(n)$ shown in formula (1), the corresponding node will be selected as the $\mathrm{CH}$ in the $r_{t h}$ round [8].

$$
T(n)= \begin{cases}\frac{p}{N-p \times\left(r \bmod \frac{N}{p}\right)} & \text { if } n \in G \\ 0 & \text { otherwise }\end{cases}
$$

When all the CHs are formed in this round, each $\mathrm{CH}$ will inform all the other $\mathrm{CHs}$ about the broadcast message of its information. If the broadcast messages of $\mathrm{CH} x$ is received by $\mathrm{CH} y$ and the signal strength exceeds a certain threshold, $\mathrm{CH} y$ will determine $\mathrm{CH} x$ as its neighbor $\mathrm{CH}$.

All the sensor nodes will join in the corresponding cluster after all the $\mathrm{CHs}$ broadcast their information. When all the sensor nodes except the $\mathrm{CHs}$ receive the broadcast message, they will select the $\mathrm{CH}$ with strongest signal as the final $\mathrm{CH}$, namely joining in the cluster. Then all the sensor nodes transmit message including their residual energy $e$ through CSMA channel, which will be used in step (3) to select cooperative nodes.

\section{(2) Routing}

$\mathrm{CH}$ calculates the minimum inter-cluster energy consumption per bit $E$ for cooperative transmission according to the information received from the other sensor nodes in the same cluster and the neighbor $\mathrm{CH}$ listed in step (1), and then obtains the number of cooperative nodes $M_{t}$. The detailed analysis will be described in Section 3 .

The routing table of $\mathrm{CH}$ is mainly composed of the ID of sink node, the ID of all the adjacent CHs and the energy consumption $E$ for cooperative transmission to each of them at the beginning. Afterwards, each $\mathrm{CH}$ exchanges the routing table with all the adjacent $\mathrm{CHs}$ and upgrades the routing table. After several times of exchanging and upgrading, each $\mathrm{CH}$ will obtain the optimal path to the sink node based on a certain routing algorithm. To simplify the system complexity, this paper chooses the most simple and practical routing algorithm Dijkstra.

(3) Scheduling

The actual energy consumption $E$ for cooperative inter-cluster transmission can be determined by the routing path to the sink node, and then $M_{t}$ sensor nodes are selected as virtual transmitting antenna array. The rule of selecting the $M_{t}$ cooperative nodes is to select the largest $M_{t}$ nodes with value of e mentioned in step (1) in order, the reason of which is to balance the residual energy of the sensor nodes within the 
cluster to delay the first node death time. Finally, the $\mathrm{CH}$ generates a TDMA scheduling message and broadcasts it to the sensor nodes within the cluster, and the cooperative sensor nodes will transmit the data according to the scheduling information.

(4) Data transmission

In data transmission phase, intra-cluster sensor nodes will transmit data according to the scheduling information received. It is notable that each sensor node can transmit data in the corresponding time slot allocated by TDMA scheduling and go into sleep mode in the other time slots in a frame to save energy.

The detailed data transmission procedure is described as follows: 1) Data is transmitted from sensor node to the $\mathrm{CH}$ within the cluster; 2) Data processed by the $\mathrm{CH}$ then is broadcasted to the $M_{t}$ intra-cluster cooperative sensor nodes; 3 ) Cooperative sensor nodes code the data through STBC after receiving the broadcast data, and then transmit together to the next $\mathrm{CH}$; 4) The next $\mathrm{CH}$ receives and decodes the data to restore the original data. 5) Repeat steps (5) (6) until the data reach to the sink node.

The procedure from step (1) to step (4) above would execute in order each round until there is no data transmitted or all the sensor nodes are dead, or the round will reach to the given threshold.

\section{ENERGY CONSUMPTION ANALYSIS}

In data transmission phase, the total intra-cluster energy consumption for broadcasting data should be considered first. We assume the transmit energy consumption for broadcasting data to the sensor nodes within the cluster as $E_{p 1}$. The radius of cluster $d_{m}$ is much smaller than the inter-cluster distance $d$, so the data transmission within the cluster will have a much lower error probability than that in the inter-cluster transmission. $E_{p 1}$ can be assumed to be constant because the intra-cluster transmission can be approximately regarded as error-free [9]. When $M_{t}$ sensor nodes try to receive data, the total intra-cluster energy consumption for broadcasting data can be expressed as:

$$
E_{1}=E_{t 1}+E_{c t}+M_{t} E_{c r}
$$

Where, $E_{t 1}=(1+\alpha) E_{p 1}$ is the energy consumption of power amplifier of $\mathrm{CH}$, which is dependent on the transmit energy consumption $E_{p 1} . \alpha=\zeta / \eta-1$ denotes the transmit efficiency with $\eta$ the drain efficiency of the RF power amplifier and $\zeta$ the Peak-to-Average Ratio (PAR) dependent on the modulation scheme and the associated constellation size $b . E_{c t}$ and $E_{c r}$ are the transmitter and the receiver circuit energy consumptions per bit, respectively.

Then we consider the inter-cluster cooperative transmission. The distances of all the sensor nodes at the transmitter side to the next $\mathrm{CH}$ can be approximately regarded as the inter-cluster distance due to $d \gg>$ $d_{m}$. Therefore, the total energy consumption of the cooperative transmission $E_{2}$ is shown as:
$E_{2}=E_{t 2}+E_{c r}+M_{t} E_{c}$

Where, $E_{t 2}$ is the total transmit energy consumption which can be calculated according to the link budget relationship [11]. Specifically, when the channel experiences a path loss with factor $k$ we have:

$E_{t 2}=(1+\alpha) \frac{(4 \pi)^{2} d^{k} M_{l} N_{f}}{G_{t} G_{r} \lambda^{2}} E_{r}$

In formula (4), the value of $k$ can be different according to different environment, for example, $k=2$ for free space propagation model, and $k=3 \sim 5$ for urban model with cellular shadows. $E_{r}$ is the average energy consumption per bit required at the receiver for a given bit error rate (BER) requirement. $d$ denotes the average transmit distance, i.e. inter-cluster distance. $G_{t}$ and $G_{r}$ is the transmitter and receiver antenna gains, respectively. $\lambda$ is the carrier wavelength. $M_{l}$ is the link margin compensating the hardware process variations and other additive background noise or interference. $N_{f}$ is the receiver noise figure. $E_{r}$ for virtual MISO can be refined as follows when $b \geq 2$ according to this paper [2]:

$E_{r} \leq \frac{2}{3}\left(\frac{P_{b}}{4}\right)^{-\frac{1}{M_{t}}} \frac{2^{b}-1}{b^{\frac{1}{M_{t}}+1}} M_{t} N_{0}$

By approximating the upper bound as equality, we have

$E_{r}=\frac{2}{3}\left(\frac{P_{b}}{4}\right)^{-\frac{1}{M_{t}}} \frac{2^{b}-1}{b^{\frac{1}{M_{t}}+1}} M_{t} N_{0}$

Combining the expression above with formula (4), we obtain

$E_{t 2}=\frac{2}{3}(1+\alpha)\left(\frac{P_{b}}{4}\right)^{-\frac{1}{M_{t}}} \frac{2^{b}-1}{b^{\frac{1}{M_{t}}+1}} M_{t} N_{0} \frac{(4 \pi)^{2} d^{k} M_{l} N_{f}}{G_{t} G_{r} \lambda^{2}}$ (5)

In conclusion, the total energy consumption per bit $E$ can be expressed as

$$
\begin{aligned}
& E=E_{1}+E_{2}=E_{t 1}+E_{t 2}+\left(M_{t}+1\right)\left(E_{c t}+E_{c r}\right) \\
& =(1+\alpha) E_{p 1} \\
& +\frac{2}{3}(1+\alpha)\left(\frac{P_{b}}{4}\right)^{-\frac{1}{M_{t}}} \frac{2^{b}-1}{b^{\frac{1}{M_{t}}+1}} M_{t} N_{0} \frac{(4 \pi)^{2} d^{k} M_{l} N_{f}}{G_{t} G_{r} \lambda^{2}} \\
& +\left(M_{t}+1\right)\left(E_{c t}+E_{c r}\right)
\end{aligned}
$$

To simplify the network complexity, we assume that the BPSK modulation is adopted in our strategy, i.e. constellation size $b=2$, and then the value of $\alpha$ is determined as well. The first invariant variables of formula (6) are given as follows:

$C_{0}=E_{t 1}=(1+\alpha) E_{p 1}=$ constant

The transmitting and receiving circuit energy consumption can be approximately regarded as constant 
[4], so the second invariant variables of formula (6) are given as follows:

$$
C_{1}=E_{c t}+E_{c r}=\text { constant }
$$

In addition, parameters such as $N_{0}, \lambda, M_{l}, N_{f}, G_{t}, G_{r}$ are fixed when the network is initialized, so the third invariant variables of formula (6) are given as follows:

$C_{2}=\frac{2}{3}(1+\alpha) \frac{2^{b}-1}{b} N_{0} \frac{(4 \pi)^{2}}{\lambda^{2}} \frac{M_{l} N_{f}}{G_{t} G_{r}}=$ constant

Combining $C_{0}, C_{1}$ and $C_{2}$ with formula (6), we have

$E=C_{0}+C_{1}\left(M_{t}+1\right)+C_{2} d^{k} M_{t}\left(\frac{4}{P_{b} b}\right)^{\frac{1}{M_{t}}}$

For a particular inter-cluster transmission, $d$ and $k$ are constant, and BER $P_{b}$ given is fixed as well when the network is initialized. Therefore formula (7) is the function of variable $M_{t}$, and there is a minimum value of $E$. Calculating the derivation of $E$ with $M_{t}$, we obtain

$$
\frac{d E}{d M_{t}}=C_{1}+C_{2} d^{k}\left(\frac{4}{P_{b} b}\right)^{\frac{1}{M_{t}}}\left[1-\frac{1}{M_{t}} \ln \left(\frac{4}{P_{b} b}\right)\right] .
$$

Making the expression above equal to 0 , we obtain

$$
d^{k}\left(\frac{4}{P_{b} b}\right)^{\frac{1}{M_{t}}}\left[\frac{1}{M_{t}} \ln \left(\frac{4}{P_{b} b}\right)-1\right]=\frac{C_{1}}{C_{2}} .
$$

The optimal $M_{t}$ is $M_{\text {topt }}=f\left(d^{k}\right)$, which can be obtained by solving the expression above. For a given inter-cluster distance $d$, the minimum energy consumption can be optimized by $M_{\text {topt }}$ obtained. Then $\mathrm{CH}$ can choose the integer approaching to $M_{\text {topt }}$ as the actual $M_{t}$.

Formula (7) is the relationship between the inter-cluster energy consumption and the path, where $E$ and $d$ is the one-to-one relationship based on optimized $M_{t}$. The actual inter-cluster energy consumption can be obtained according to formula (7), thereby the optimal path from the sensor node with data transferred initially to the sink node can be determined by Dijsktra routing algorithm.

\section{SIMULATION AND ANALYSIS}

\subsection{Text and indenting}

The proposed virtual MIMO network is simulated using the model described in Figure 1. There are 100 nodes in the simulation scenario, network area is $500 \mathrm{~m} \times 500 \mathrm{~m}$, and the sink node is located at the center of the network. The initial energy of each sensor node is $1 \mathrm{~J}$, and the data with 1 bit length generated in a node randomly each round. The simulation parameters [3] are given in Table 1.

In order to validate the performance, we compare the proposed strategy of optimized cooperative MISO (OCMISO) with the strategy of LEACH, noncoopera- tive SISO (NCSISO) and random cooperative MISO (RCMISO). The strategy of LEACH is that data collected by the $\mathrm{CH}$ is directly transmitted to the sink node. The strategy of NCSISO is that data collected by the $\mathrm{CH}$ is transferred to the sink node through multi-hop transmission communication using Dijkstra routing algorithm based on the cost of distance. The strategy of RCMISO involving cooperative virtual MISO communication selects cooperative nodes randomly based on NCSISO, and the cost of the routing algorithm is the inter-cluster energy consumption calculated by formula (6). The proposed strategy of OCMISO is to select optimized cooperative nodes and find the optimal path to the sink node based on the cost of the minimum inter-cluster energy consumption.

Table 1. Simulation parameters setting

\begin{tabular}{ll}
\hline $\mathrm{B}=10 \mathrm{kHz}$ & $\mathrm{N}_{0}=-171 \mathrm{dBm} / \mathrm{Hz}$ \\
$\mathrm{f}_{\mathrm{c}}=2.5 \mathrm{GHz}$ & $\mathrm{k}=2 \sim 5$ \\
$\mathrm{M}_{\mathrm{l}}=40 \mathrm{~dB}$ & $\lambda=0.12 \mathrm{~m}$ \\
$\mathrm{~N}_{\mathrm{f}}=10 \mathrm{~dB}$ & $\zeta=1$ \\
$\mathrm{G}_{\mathrm{t}} \mathrm{G}_{\mathrm{r}}=5 \mathrm{dBi}$ & $\mathrm{P}_{\mathrm{ct}}=84 \mathrm{~mW}$ \\
$\eta=0.35$ & $\mathrm{P}_{\mathrm{cr}}=112 \mathrm{~mW}$ \\
$\mathrm{P}_{\mathrm{b}}=10^{-3}$ & $\mathrm{R}_{\mathrm{b}}=10 \mathrm{~kb} / \mathrm{s}$
\end{tabular}

Comparison of the total energy consumption of the network with the round is shown in Figure 2. Total energy consumption of the four algorithms is on the rise with the round increasing. In the same round, the relationship of the four kinds of algorithm about the total energy consumption is LEACH $>$ NCSISO > RCMISO > OCMISO. LEACH is a classical WSN data transmission strategy, where data integrated to the $\mathrm{CH}$ is directly transmitted to the sink node, so the energy consumption will become very large because of the long distance between the $\mathrm{CH}$ and the sink node. Multi-hop transmission mode is introduced to NCSISO to break down the long distance into many short distances, largely reducing the transmission energy consumption, has become the mainstream in the WSN research, but the routing algorithm is based on the shortest path without considering energy consumption. RCMISO further reduces the energy consumption compared with NCSISO by using virtual MIMO communication, but the inter-cluster energy consumption is not minimized because of the cooperative nodes selected randomly. Considering the circuit and the transmission energy consumption of the sensor nodes comprehensively, OCMISO optimizes the number of the cooperative nodes compared with RCMISO to minimize the inter-cluster energy consumption, so the total energy consumption of the network become minimum. Similarly, for the same value of the energy consumption in Figure 2, the round of OCMISO is the largest, which means that OCMISO can prolong the life cycle of the network because of 


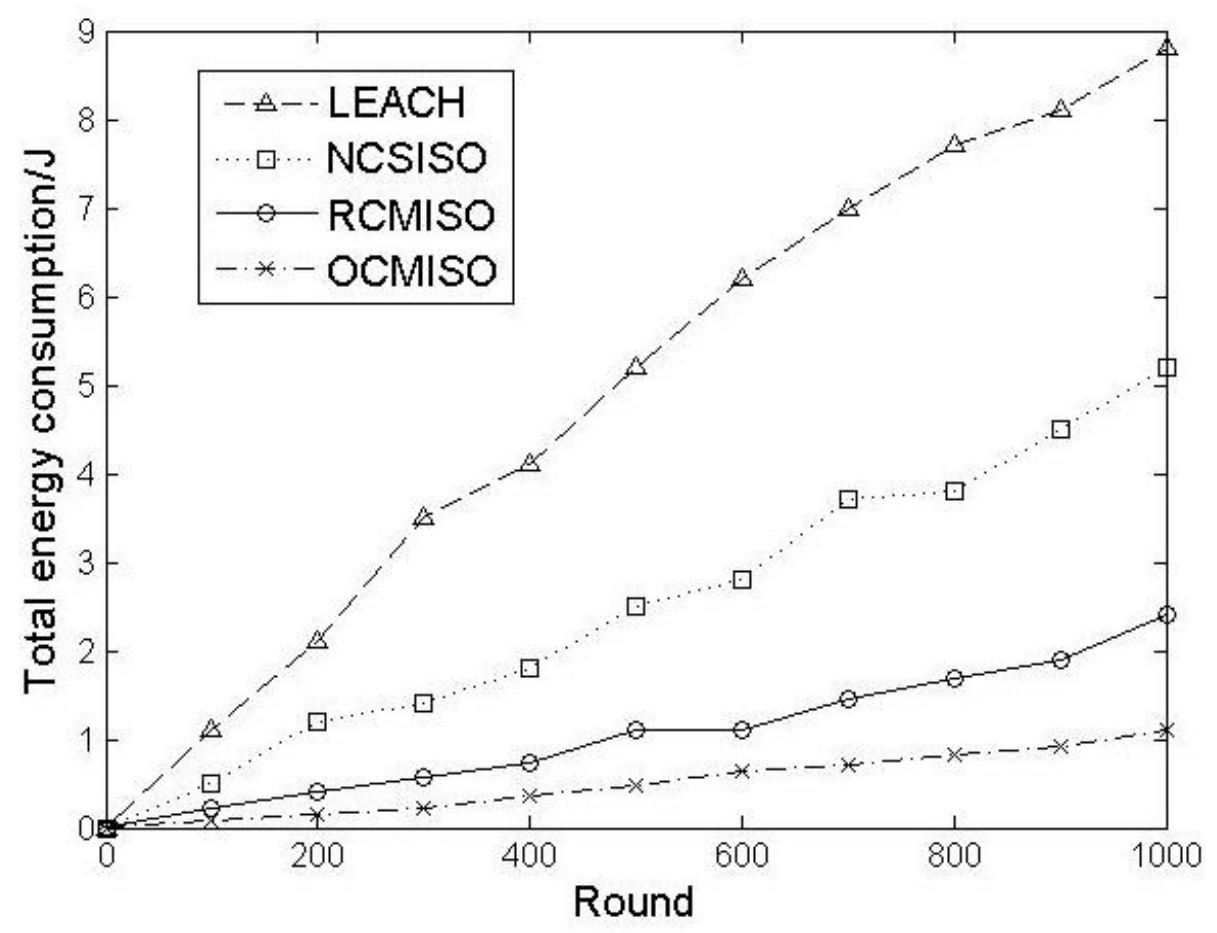

Figure 2. Total energy consumption of the network.

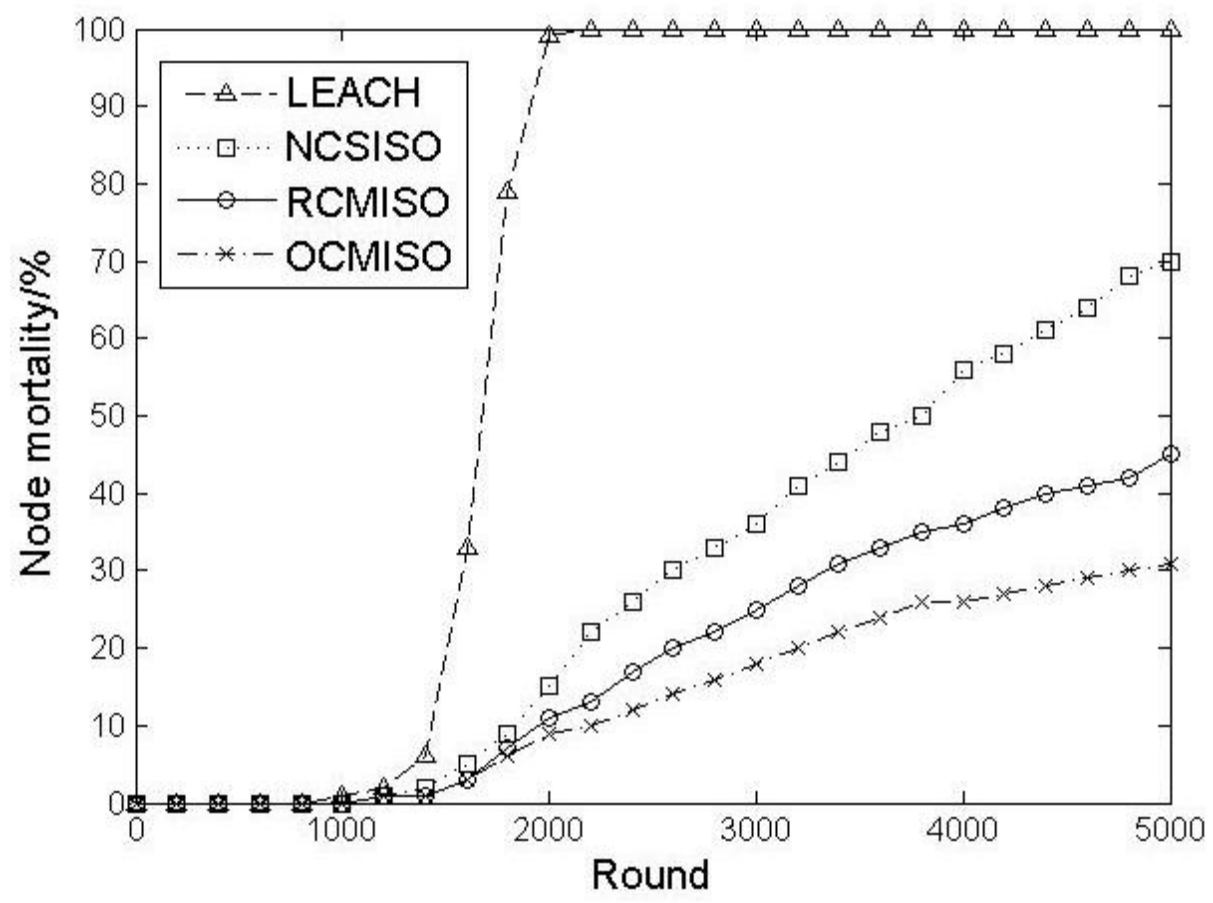

Figure 3. Node mortality of the network

more energy conservation. 
Comparison of the node mortality with the round is shown in Figure 3. As can be seen from Figure 3, the dead node percentages of LEACH, NCSISO, RCMISO and OCMISO are about $100 \%, 22 \%, 14 \%$ and $10 \%$ respectively in 2200 round. Therefore, communication involving multi-hop and cooperative mode will prolong the life cycle of the network by using its cooperative diversity gain to save more transmission energy consumption effectively.

\section{CONCLUSIONS}

This paper models the WSN with virtual MIMO communication by considering the characteristics of the limited energy of the WSN. A multi-hop cooperative transmission strategy is proposed based on the minimum inter-cluster energy consumption, transforming the energy consumption problem into the distance problem by optimizing of the number of the cooperative nodes, and obtains the optimal path to the sink node. The proposed strategy enlarges the range of communication and decreases the routing complexity. The simulation results show that the proposed strategy represents better performance of energy conservation with round or inter-cluster distance.

\section{ACKNOWLEDGEMENTS}

This work was supported by the Project of Education and Scientific Research of Young Teacher of Fujian (GN: JA14233) and the Young Scientists Fund of the National Natural Science Foundation of China (GN: 61202013).

\section{REFERENCES}

[1] Paulraj A, Nabar R. \& Gore D. 2003. Introduction to space-time wireless communications. Cambridge university press.

[2] Cui S, Goldsmith A J. \& Bahai A. 2004. Energy-efficiency of MIMO and cooperative MIMO techniques in sensor networks. IEEE Journal on Selected Areas in Communications 22(6): 1089-1098.

[3] Cui S, Goldsmith A J. \& Bahai A. 2005. Energy-constrained modulation optimization. IEEE Transactions on Wireless Communications 4(5): 2349-2360.

[4] [Hussain S, Azim, \& Park J H. 2009. Energy efficient virtual MIMO communication for wireless sensor networks. Telecommunication Systems 42(1-2): 139-149.

[5] Xue J, Zhang T. \& Yan Y, et al. 2013. Cooperation-based Ant Colony Algorithm in WSN. Journal of Networks 8(4): 939-946.

[6] Qin Z C, Zhou Z. \& Zhao X C, etal. 2013. Cooperative transmission scheme based on GSC for cognitive wireless sensor network. Journal on Communications 34(9): 158-165.
[7] Qiu Y Z, Shen J. \& Dong S L, etal. 2013. Research on energy-efficient of virtual MIMO based on wireless sensor networks. Computer Engineering, 34(9): 158-165.

[8] Heinzelman W R, Chandrakasan A. \& Balakrishnan H. 2000. Energy-efficient communication protocol for wireless microsensor networks. Proceedings of the 33rd annual Hawaii international conference on System sciences 10 pp. vol. 2.

[9] Dai L, Chen W. \& Cimini L J, et al. 2009. Fairness improves throughput in energy-constrained cooperative ad-hoc networks. IEEE Transactions on Wireless Communications 8(7): 3679-3691.

[10]Li B, Wang W, Yin Q, et al. 2012. A new cooperative transmission metric in wireless sensor networks to minimize energy consumption per unit transmit distance. Communications Letters 16(5): 626-629.

[11]Proakis J G. 1995. Digital Communications. New York: McGraw-Hill. 